\title{
Use of a Minimally Invasive Cardiac Output Monitor to Optimise Haemodynamics in a Patient with Mitral Valve Disease Undergoing Cerebrovascular Surgery
}

Ali M. Al-Mashani, ${ }^{1}$ Niranjan D. Waje, ${ }^{2}$ Neeraj Salhotra, ${ }^{1}$ Samaresh Das, ${ }^{3}$ Neelam Suri, ${ }^{3}$ Rashid A. Al-Sheheimi, ${ }^{4}$ *Nilay Chatterjee ${ }^{3}$

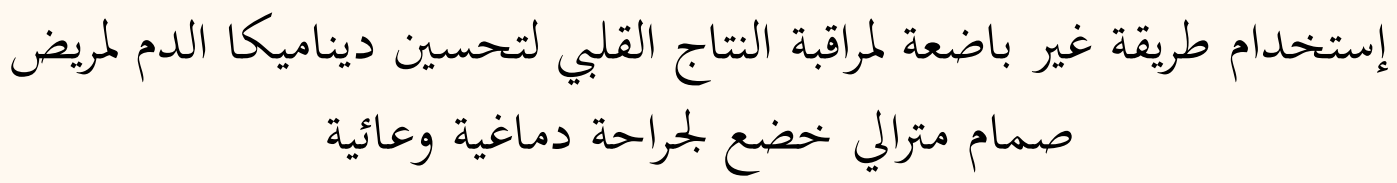

علي محاد المعشني، نيرانجان واجي، نيراج سالهوترا، ساماريتش داز، ميلان سوري، راشد أحمد الشهيمي، نيلاي شاترجي

ABSTRACT: Patients with mitral valve disease undergoing cerebrovascular surgery face increased inherent risks due to their associated cardiac comorbidities. As such, the anaesthetic management of such patients is distinctly challenging. Simultaneous consideration of both the cerebrovascular and underlying cardiac conditions determines key anaesthetic issues, as fluids and vasopressors or inotropes need to be titrated according to haemodynamic variables in order to optimise cerebral blood flow without compromising cardiac function. We report a 45-yearold female patient with mild mitral stenosis and moderate-to-severe mitral regurgitation who presented to the Khoula Hospital, Muscat, Oman, in 2016 following a ruptured anterior communicating artery aneurysm requiring urgent surgical intervention. As highlighted in this case, the VolumeView EV1000 ${ }^{\mathrm{TM}}$ (Edwards Lifesciences, Irvine, California, USA) system is a minimially invasive haemodynamic monitor that can help immensely in the perioperative management of such patients.

Keywords: Anesthesia; Cerebral Aneurysm; Mitral Valve Stenosis; Mitral Valve Regurgitation; Hemodynamics; Cardiac Output; Case Report; Oman.

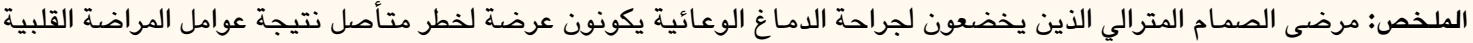

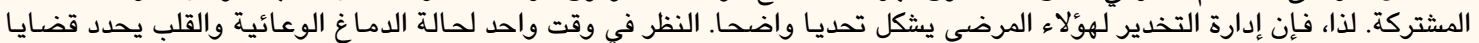

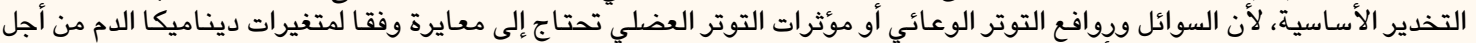

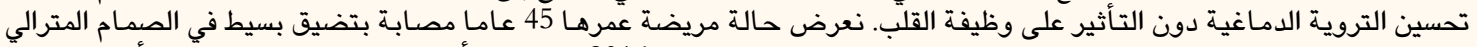

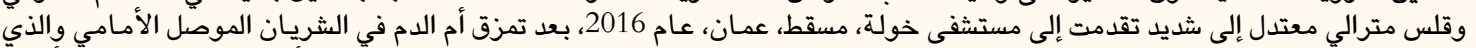

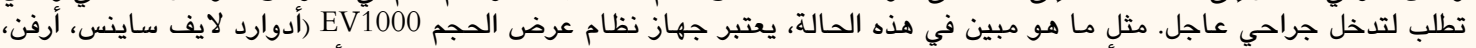

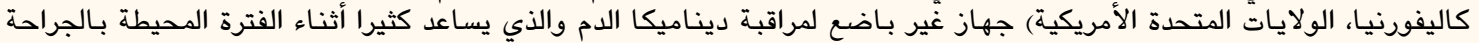
لهوزلاء المرضى. الكلمات المفتاحية: التخدير؛ أم الدم الدخية؛ تضيق الصمام المترالي؛ قلس الصمام المترالي؛ ديناميكا الدم؛ النتاج القلبي؛ تقرير حالة؛ عمان.

$\mathrm{S}$ UBARACHNOID HAEMORRHAGE SECONDARY TO a ruptured intracerebral aneurysm and the subsequent surgery for clipping of the aneurysm is associated with its own inherent risks. ${ }^{1}$ However, such intraoperative risks are increased when the patient also has valvular heart disease or an associated cardiac comorbidity. Simultaneous consideration of both the cerebrovascular and cardiac conditions is particularly challenging for anaesthesiologists as key issues in the perioperative management of haemodynamic goals are contradictory at times and require precise and continuous monitoring of cardiac output and other related variables. ${ }^{1}$ The use of the VolumeView
$\mathrm{EV}_{1000}{ }^{\mathrm{TM}}$ (Edwards Lifesciences, Irvine, California, USA) system in a neurosurgical setting has not yet been described in the literature. However, it may be a useful monitoring tool for goal-directed therapy in such cases.

The VolumeView EV1000 ${ }^{\mathrm{TM}}$ (Edwards Lifesciences) system is a minimally invasive device that measures haemodynamic parameters and guides the use of fluids and vasopressors for patient-specific goaldirected therapy. It provides pulse contour analysisderived calibrated haemodynamic parameters-including stroke volume variation (SVV), cardiac output and systemic vascular resistance (SVR) - as well as 
parameters for fluid responsiveness derived from a transpulmonary thermodilution algorithm, such as the global end-diastolic volume index (GEDVI), extravascular lung water index (EVLWI), pulmonary vascular permeability index (PVPI) and contractility (i.e. cardiac output, stroke volume, global ejection fraction and SVR). ${ }^{2}$ The physiological parameters derived by the VolumeView EV1000 ${ }^{\mathrm{TM}}$ system (Edwards Lifesciences) help to determine the precise titration of fluid and vasopressors or inotropes; moreover, its accuracy is comparable to that of more invasive monitors. ${ }^{3}$ This case report describes the anaesthetic management of a patient with mitral valve disease who underwent a craniotomy for clipping of an anterior communicating artery aneurysm. The role of a minimally invasive cardiac output monitor in achieving targeted haemodynamic goals in such patients is highlighted.

\section{Case Report}

A 45-year-old woman presented to the Khoula Hospital, a tertiary care neurosurgical centre in Muscat, Oman, in 2016 with a sudden-onset, severe, holocranial headache of seven days' duration. She had no history of vomiting, loss of consciousness or altered sensorium. However, she reported a history of shortness of breath on exertion (grade II dyspnoea) over the previous four years. On examination, she was found to be conscious, cooperative and alert, with a Glasgow coma scale score of 15 , a heart rate of 65 beats/minute and a respiratory rate of 20 breaths/ minute. Her blood pressure was $124 / 80 \mathrm{mmHg}$. A neurological examination was unremarkable and the headache symptoms were categorised as Hunt and Hess grade II.

A computed tomography (CT) scan revealed evidence of a subarachnoid haemorrhage (Fisher grade II) and an angiogram showed an anterior communicating artery aneurysm measuring $5.7 \times 6.2 \times 7.0 \mathrm{~mm}$. The A1 segment of the right anterior cerebral artery showed evidence of vasospasm. Transthoracic echocardiography revealed thickened calcific mitral valve leaflets with mild mitral stenosis (mitral valve area: $1.6 \mathrm{~cm}^{2}$ ), moderate-to-severe mitral regurgitation (vena contracta width: $0.5 \mathrm{~cm}$; effective regurgitant orifice area: $0.4 \mathrm{~cm}^{2}$ ) with an eccentric jet hugging the posterior wall of the left atrium and an ejection fraction of $50 \%$ with adequate biventricular function. The pulmonary vein flow pattern on the contralateral side showed systolic blunting without reversal and the mitral regurgitation was graded as moderate-tosevere. ${ }^{4}$ All other haematological and biochemical investigations were within normal limits. An urgent craniotomy was planned to clip the aneurysm.

In the operating room, standard monitoring equipment including electrocardiography, pulse oximetry and non-invasive blood pressure equipment was set up as per the recommendations of the American Society of Anesthesiologists. ${ }^{1}$ The baseline pre-induction blood pressure was 124/55 $\mathrm{mmHg}$. Since the patient had a high perioperative cardiac risk and a narrow therapeutic window for fluid administration, a VolumeView EV1000 ${ }^{\mathrm{TM}}$ (Edwards Lifesciences) arterial catheter with a temperature sensor was inserted in the right femoral artery and a triple lumen central venous catheter was placed in the right internal jugular vein while she was under sedation and local anaesthesia. Subsequently, the VolumeView EV1000 ${ }^{\mathrm{TM}}$ (Edwards Lifesciences) cardiac output monitor was connected. Following preoxygenation, general endotracheal anaesthesia was administered using propofol, fentanyl, sevoflurane and vecuronium. A decrease in blood pressure following induction was managed judiciously with various aliquots of ephedrine.

The anaesthesia was maintained with airoxygen-isoflurane-propofol and supplemental doses of vecuronium-fentanyl, as required. The ventilation parameters were as follows: volume-guaranteed pressure-controlled ventilation, a peak inspiratory pressure of $20 \mathrm{~cm}$, tidal volume of $\sim 450 \mathrm{~mL}$ (for $8 \mathrm{~mL} / \mathrm{kg}$ of ideal body weight) and a respiratory rate of 14 breaths/minute (for a partial pressure of carbon dioxide $\left[\mathrm{CO}_{2}\right]$ of $32-35 \mathrm{mmHg}$ ). Intraoperatively, judicious fluid administration (including lactated ringers solution and $0.9 \%$ sodium chloride) and inotrope/vasopressor therapy were strictly guided by the physiological parameters derived by the VolumeView EV1000 ${ }^{\mathrm{TM}}$ (Edwards Lifesciences) system. The perioperative haemodynamic goals consisted of a low-to-normal SVR index and an augmented cardiac output. The use of colloids was avoided entirely.

Optimum brain relaxation was achieved with 20\% mannitol and controlled hyperventilation to maintain end-tidal $\mathrm{CO}_{2}$ at 30-32 mmHg. Systolic blood pressure was allowed to fall to $95 \mathrm{mmHg}$ immediately before the clipping of the aneurysm. A temporary clip application to the feeding arteries of the aneurysm was not required during the surgery. Following the application of a permanent clip, blood pressure was augmented for a target mean arterial pressure (MAP) of $110 \mathrm{mmHg}$ as the maximum upper limit using titrated doses of a noradrenaline infusion. However, this was associated with a significant fall in cardiac output, an increase in SVR index, a simultaneous drop in oxygen saturation (from $100 \%$ to $91 \%$ ) with a rise in 
Table 1: Haemodynamic parameters derived by a minimally invasive cardiac output monitor* during an aneurysm clipping surgery for a patient with mitral valve disease

\begin{tabular}{|c|c|c|c|c|c|c|}
\hline Time point & SVV & $\begin{array}{c}\text { GEDVI in } \\
\mathrm{mL} / \mathrm{m}^{2}\end{array}$ & $\begin{array}{l}\text { EVLWI in } \\
\mathrm{mL} / \mathrm{kg}\end{array}$ & PVPI & $\begin{array}{c}\mathrm{CO} \text { in } \mathrm{L} / \\
\text { minute }\end{array}$ & $\begin{array}{l}\text { SVRI in dynes } \\
\text { second } / \mathrm{cm}^{5}\end{array}$ \\
\hline Immediately post-induction & 22 & 690 & 9.2 & 2.3 & 4.8 & 1,460 \\
\hline $\begin{array}{l}\text { Immediately before clipping of } \\
\text { the aneurysm }\end{array}$ & 28 & 675 & 8.7 & 2.4 & 4.5 & 1,123 \\
\hline $\begin{array}{l}\text { Post-aneurysm clipping with } \\
\text { noradrenaline infusion }\end{array}$ & 17 & 790 & 14.2 & 3.3 & 2.8 & 3,460 \\
\hline $\begin{array}{l}\text { Post-aneurysm clipping with } \\
\text { dobutamine and noradrenaline } \\
\text { infusions }\end{array}$ & 13 & 689 & 11.7 & 2.6 & 4.8 & 2,043 \\
\hline
\end{tabular}

$S V V=$ stroke volume variation; GEDVI = global end-diastolic volume index; EVLWI = extravascular lung water index; PVPI = pulmonary vascular permeability index; $C O=$ cardiac output; SVRI = systemic vascular resistance index.

*Using the VolumeView EV1000 ${ }^{T M}$ (Edwards Lifesciences, Irvine, California, USA) system.

peak airway pressure (from 19 to $24 \mathrm{~cm}$ ) and increases in EVLWI and GEDVI [Table 1].

At this point, low-dose dobutamine was administered at $5 \mu \mathrm{g} / \mathrm{kg} /$ minute to augment forward flow with concomitant titration of the noradrenaline infusion in order to attain an acceptable MAP. ${ }^{5}$ In this way, the patient's blood pressure increased to 160/82 mmHg (mean: $108 \mathrm{mmHg}$ ). The SVR index was relatively low after the induction of the anaesthesia, possibly due to anaesthesia-mediated vasodilation; the baseline SVR index was 1,680 dynes.second $/ \mathrm{cm}^{5}$, which fell to approximately 1,460 dynes.second $/ \mathrm{cm}^{5}$ after induction and then 1,100-1,300 dynes.second $/ \mathrm{cm}^{5}$ before the clipping of the aneurysm (normal range: 2,000-2,400 dynes.second $/ \mathrm{cm}^{5}$ ). Throughout the course of the surgery, any changes in the haemodynamic variables were continuously monitored, including heart rate, MAP, SVV, cardiac output and SVR index. The extubation of the patient and the postoperative course were uneventful. On the third postoperative day, a repeat CT scan was essentially normal, with evidence that the subarachnoid haemorrhage was resolving. There was no indication of a cerebral infarction following the surgery.

\section{Discussion}

The presence of valvular cardiac disease increases the anaesthetic and perioperative risks of noncardiac surgery. ${ }^{1}$ There are three key haemodynamic targets in the perioperative management of a cerebral aneurysm. Firstly, haemodynamic perturbations should be prevented when inducing anaesthesia and deliberate hyperventilation or hypocania should be avoided before the opening of the dura, as both of these circumstances may cause the aneurysm to rupture secondary to sudden changes in the transmural pressure gradient. ${ }^{6,7}$ Secondly, cerebral perfusion pressure should be preserved, including the augmentation of arterial blood pressure during temporary clip application to curtail the impact of ischaemia in regions of the brain located upstream to the clamped artery and the suppression of cerebral metabolism with titrated doses of propofol or other volatile anaesthetic agents. Finally, the arterial blood pressure should be raised (systolic blood pressure: 160-200 mmHg; MAP: >100 mmHg) following permanent clip application, thereby preventing ischaemia in vasospastic segments. ${ }^{6,7}$ Formerly, triple-H therapy (i.e. hypertension, hypervolaemia and haemodilution) was a popular technique for managing vasospasm and related complications; however, hypervolaemia and haemodilution have since fallen out of favour due to their inherent complications, although induced hypertension (systolic blood pressure of 160-200 mmHg once the aneurysm is secured) is still routinely employed to ensure adequate cerebral blood flow. ${ }^{7}$

Cardiac output monitoring is an important tool in goal-directed therapy for critically ill patients. ${ }^{3}$ While a pulmonary artery catheter was formerly the goldstandard monitoring approach, its use is associated with various complications and increased mortality; accordingly, it has been progressively replaced by less invasive monitoring techniques. ${ }^{8-10}$ A reliable cardiac output monitor, ideally a minimally invasive one, could be of immense use for monitoring patients with cardiac comorbidities undergoing cerebrovascular surgery. Due to her pre-existing heart condition, the patient in the current case required precise monitoring of her left ventricular function. In addition, flow augmentation management had significant limitations due to the mild mitral stenosis and moderate-to-severe mitral regurgitation. Moreover, increasing the SVR with noradrenaline to achieve the target MAP adversely affected the cardiac output, regurgitant fraction and myocardial function because of the concomitant mitral regurgitation. 
On the other hand, augmenting cardiac output using inotropes (i.e. dobutamine) could have potentially caused excessive tachycardia and worsened the transmitral pressure gradient and pulmonary congestion (due to increased EVLWI). ${ }^{5}$ This could have led to a deterioration in oxygenation. Arguably, common practices to augment cerebral blood flow in vasospastic areas without compromising myocardial function and oxygenation are not themselves free of complications. ${ }^{7}$ As the mitral stenosis was mild, it was hypothesised that the patient would be better able to tolerate a higher heart rate rather than increased SVR. Hence, the aim was to perfuse the patient so as to augment cardiac output without excessively increasing the afterload, thus maintaining a low-to-normal SVR index and avoiding tachycardia for a target heart rate of 80-90 beats/minute. This was expected to maintain the forward flow and limit the transmitral pressure gradient, thus minimising the regurgitant volume (i.e. a lower EVLWI, GEDVI and SVV). As such, a reliable monitoring tool was needed to achieve these haemodynamic targets without adversely affecting cardiac output, regurgitant fraction and myocardial function.

Transoesophageal echocardiography is another useful tool for the perioperative monitoring of cardiac output in neurosurgical patients. ${ }^{11}$ It has been validated in comparison to pulmonary artery catheters with a good outcome. ${ }^{12}$ However, transesophageal echocardiography requires a skilled operator and its use is limited by the cost and availability of the equipment. The VolumeView EV1000 ${ }^{\mathrm{TM}}$ (Edwards Lifesciences) system requires both central and arterial cannulation via the internal jugular and femoral artery, respectively. Central venous pressure does not adequately reflect the preload status and is therefore unsuitable for predicting the ventricular response to fluid loading; the GEDVI, a volumetric preload variable, more adequately reflects cardiac preload changes. ${ }^{13} \mathrm{~A}$ supranormal GEDVI and EVLWI indicates excessive fluid balance with pulmonary congestion, whereas a PVPI of $>3$ indicates increased pulmonary vascular permeability and thereby potentially differentiates cardiogenic oedema from acute respiratory distress syndrome. $^{13,14}$ An EVLWI of $>10$ is associated with increased morbidity and mortality. ${ }^{14}$ In the present patient, the use of a vasopressor to increase the MAP after clipping resulted in a significant fall in cardiac output, with a concomitant increase in volume parameters (i.e. GEDVI and EVLWI) which suggested an increase in the regurgitant fraction. This was instantly recognised by the cardiac output monitor, enabling prompt use of an inodilator to augment the forward flow.
Intraoperatively, due to the mild mitral stenosis and moderate-to-severe mitral regurgitation, the current patient required a comparatively high heart rate with a low-to-normal SVR index to augment the forward flow. Among the different options available to augment cerebral perfusion following clipping of the aneurysm, the continuous infusion of noradrenaline (a $\beta$ - and $\alpha$-agonist) was the preferred choice over phenylephrine (a pure $\alpha$-agonist). This was in view of the ability of noradrenaline to prevent the reflex bradycardia associated with a raised MAP. ${ }^{5}$ However, the physiological parameters worsened (i.e. increased SVR index and EVLWI with decreased cardiac output) following the augmentation of the MAP using a noradrenaline infusion alone. Initiating titrated doses of dobutamine resulted in a further increase in heart rate with a drop in SVR index. This was deemed appropriate in order to augment cerebral perfusion to the targeted level and was subsequently reflected in the physiological parameters (i.e. a decreased SVR index and EVLWI with increased cardiac output). The fall in the SVR index probably resulted in a reduction in the regurgitant fraction of the mitral regurgitation and augmentation of the cardiac output following the dobutamine infusion. Although temporary clipping was not necessary, dobutamine and noradrenaline infusions were titrated to achieve augmented perfusion as well as an appropriate MAP postoperatively.

\section{Conclusion}

Conflicting haemodynamic goals can complicate the anaesthetic management of patients with mitral valve diseases undergoing surgical clipping of a cerebral aneurysm. Minimally invasive cardiac output monitoring devices, such as the VolumeView $\mathrm{EV}_{1000}{ }^{\mathrm{TM}}$ (Edwards Lifesciences) system, are useful in such situations to aid in the accurate titration of fluids and inotropes/vasopressors according to myocardial performance, thus greatly enhancing patient safety while under anaesthesia.

\section{References}

1. Fleisher LA, Beckman JA, Brown KA, Calkins H, Chaikof E, Fleischmann KE, et al. ACC/AHA 2007 guidelines on perioperative cardiovascular evaluation and care for noncardiac surgery: Executive summary - A report of the American College of Cardiology/American Heart Association Task Force on practice guidelines. Circulation 2007; 116:1971-96. doi: 10.1161/CIRCULATIONAHA.107.185700.

2. Kiefer N, Hofer CK, Marx G, Geisen M, Giraud R, Siegenthaler N, et al. Clinical validation of a new thermodilution system for the assessment of cardiac output and volumetric parameters. Crit Care 2012; 16:R98. doi: 10.1186/cc11366. 
3. Mehta Y, Arora D. Newer methods of cardiac output monitoring. World J Cardiol 2014; 6:1022-9. doi: 10.4330/wjc. v6.19.1022.

4. Lancellotti P, Moura L, Pierard LA, Agricola E, Popescu BA, Tribouilloy C, et al. European Association of Echocardiography recommendations for the assessment of valvular regurgitation: Part 2 - Mitral and tricuspid regurgitation (native valve disease). Eur J Echocardiogr 2010; 11:307-32. doi: 10.1093/ejechocard/ jeq031.

5. Overgaard CB, Dzavík V. Inotropes and vasopressors: Review of physiology and clinical use in cardiovascular disease Circulation 2008; 118:1047-56. doi: 10.1161/CIRCULATIONA HA. 107.728840

6. Priebe HJ. Aneurysmal subarachnoid haemorrhage and the anaesthetist. Br J Anaesth 2007; 99:102-18. doi: 10.1093/bja/aem119.

7. Pomg RP, Lam AM. Anesthetic management of cerebral aneurysm surgery. In: Cottrell JE, Young WL, Eds. Cottrell and Young's Neuroanesthesia, 5th ed. Philadelphia, Pennsylvania, USA: Mosby Elsevier, 2010. Pp. 218-46.

8. Robin ED. Death by pulmonary artery flow-directed catheter: Time for a moratorium? Chest 1987; 92:727-31. doi: 10.1378/ chest.92.4.727.

9. Connors AF Jr, Speroff T, Dawson NV, Thomas C, Harrell FE Jr, Wagner $\mathrm{D}$, et al. The effectiveness of right heart catheterization in the initial care of critically ill patients: SUPPORT investigators. JAMA 1996; 276:889-97. doi: 10.1001/jama.1996.0354 0110043030
10. Bendjelid K, Marx G, Kiefer N, Simon TP, Geisen M, Hoeft A, et al. Performance of a new pulse contour method for continuous cardiac output monitoring: Validation in critically ill patients. Br J Anaesth 2013; 111:573-9. doi: 10.1093/bja/aet116.

11. Chatterjee N, Koshy T, Misra S, Suparna B. Changes in left ventricular preload, afterload, and cardiac output in response to a single dose of mannitol in neurosurgical patients undergoing craniotomy: A transesophageal echocardiographic study. J Neurosurg Anesthesiol 2012; 24:25-9. doi: 10.1097/ ANA.0b013e3182338b11.

12. Perrino AC Jr, Harris SN, Luther MA. Intraoperative determination of cardiac output using multiplane transesophageal echocardiography: A comparison to thermodilution. Anesthesiology 1998; 89:350-7.

13. Marik PE, Cavallazzi R. Does the central venous pressure predict fluid responsiveness? An updated meta-analysis and a plea for some common sense. Crit Care Med 2013; 41:1774-81. doi: 10.1097/CCM.0b013e31828a25fd.

14. Jozwiak M, Teboul JL, Monnet X. Extravascular lung water in critical care: Recent advances and clinical applications. Ann Intensive Care 2015; 5:38. doi: 10.1186/s13613-015-0081-9. 